\title{
Pediatric Glaucoma in a University Hospital
}

\author{
Mohamad Dakroub ${ }^{1}$, Diana Khair ${ }^{2}$, Bahaa' Noureddine ${ }^{3}$, Christiane Al-Haddad $^{4}$
}

\begin{abstract}
Aims and objectives: This study aimed at describing the characteristics and outcomes of pediatric glaucoma patients in a university hospital in Lebanon and comparing them between primary congenital glaucoma (PCG) and secondary glaucoma.

Materials and methods: Sixty-four eyes of 40 pediatric glaucoma patients with a follow-up of 1 year or more were reviewed. Parameters were studied for both PCG and secondary glaucoma groups. Three major outcomes were considered: intraocular pressure (IOP) control, final bestcorrected visual acuity (BCVA), and the proportion of failed angle surgeries.

Results: Sixty-four eyes were included divided into 29 PCG and 35 secondary glaucoma eyes, followed up for a mean of $6.6 \pm 4.7$ years. Eyes with PCG presented earlier, with a higher initial IOP and underwent earlier surgery than their secondary glaucoma counterparts. Although $85.9 \%$ of all eyes achieved good IOP control, $44.4 \%$ of them had a BCVA $\geq 20 / 50$ with PCG having a better visual prognosis. Secondary glaucoma eyes needed more medications at the end of their follow-up. In this group, it was shown that a later age at first surgery, unilateral disease, and multiple surgeries were associated with a BCVA $<20 / 50$.
\end{abstract}

Conclusion: Patients with PCG presented earlier and with a higher IOP than secondary glaucoma; they were also operated on earlier. Both groups demonstrated good IOP control at the end of the follow-up period with PCG eyes having a superior visual outcome. Secondary glaucoma eyes required a higher number of medications at their final visit. In this population, multiple surgeries, unilateral disease, and a delayed first surgery conferred a poorer visual prognosis.

Clinical significance: Data regarding the characteristics of pediatric glaucoma, especially in the Middle East, are scarce. Our study sheds the light on the presentation of different types of pediatric glaucoma and their outcomes namely IOP control and visual acuity.

Keywords: Congenital glaucoma, Glaucoma surgery, Intraocular pressure, Pediatric glaucoma, Retrospective chart review, Visual acuity.

Journal of Current Glaucoma Practice (2021): 10.5005/jp-journals-10078-1291

\section{INTRODUCTION}

Pediatric glaucoma is a relatively rare condition that frequently results in visual impairment. ${ }^{1}$ It is usually classified as primary, secondary, or acquired. ${ }^{1}$ The primary form includes two major categories depending on the age of onset. It is termed primary congenital glaucoma (PCG) if it presents in the first 4 years of life, and juvenile primary open-angle glaucoma if it occurs after that age. ${ }^{2}$ The primary congenital form is the most common form of pediatric glaucoma and is also the most responsive to treatment. ${ }^{2-6}$ It is more common in males and accounts for $0.01-0.04 \%$ of blindness. ${ }^{7}$ Secondary glaucoma is frequently due to congenital conditions (such as aniridia and aphakia) or various syndromic disorders, whereas acquired glaucoma typically arises from non-congenital factors such as inflammation and drugs.,

Several studies have published data on the epidemiologic and prognostic factors of PCG. It is 4 times more common in the Middle East than the West, and in Saudi Arabia, it is the cause of $16 \%$ of childhood blindness. ${ }^{8}$ This could be attributed to the increased proportion of consanguineous marriages in this region of the world. ${ }^{9}$ A study carried out on the demographics of congenital glaucoma in the West Bank and Gaza Strip showed that $49 \%$ of patients had consanguineous parents. ${ }^{10}$ Additionally, the incidence of congenital glaucoma and its genetic mutations vary by ethnicity; mutations in the cytochrome P450 gene CYP1B1 were found in $50 \%$ of patients affected with PCG worldwide. ${ }^{11}$ However, this frequency varies in different populations. Our group showed that the percentage of CYP1B1 mutations in the Lebanese population was $33 \%{ }^{12}$ while in neighboring countries such as Saudi Arabia and Israel, it was $100 \%{ }^{13}$ and $80 \%,{ }^{14}$ respectively. This is probably due to the genetic heterogeneity of the Lebanese population. ${ }^{12}$
${ }^{1-4}$ Department of Ophthalmology, American University of Beirut Medical Center, Beirut, Governorate, Lebanon

Corresponding Author: Christiane Al-Haddad, Department of Ophthalmology, American University of Beirut Medical Center, Beirut, Governorate, Lebanon, Phone: +9613982997, e-mail: ca12@aub.edu.lb How to cite this article: Dakroub M, Khair D, Noureddine B, et al. Pediatric Glaucoma in a University Hospital. J Curr Glaucoma Pract 2021;15(1):8-13.

Source of support: Nil

Conflict of interest: None

The prognosis of the disease seems to be linked to several factors including age at initial presentation, time of first surgery, the extent of optic nerve injury, presence of other ocular comorbidities, corneal size, and the development of anisometropia/amblyopia. ${ }^{15}$ Despite all the above variables, a study on 25 Arab-Bedouin children with PCG in 2005 reported presenting intraocular pressure (IOP) to be the only independent predictor of failure of treatment when success was measured as IOP $>5$ and $<21 \mathrm{~mm} \mathrm{Hg}$ at the end of a 2-year period, off medication, regardless of the number of procedures they underwent. ${ }^{16}$

The visual prognosis of PCG seems to be satisfactory with $52-79 \%$ of patients having a final visual acuity of $20 / 50$ or better. ${ }^{17}$ Other types of glaucomas including secondary glaucoma seem to have worse prognosis. ${ }^{17}$ Factors that confer a bad visual prognosis include neonatal onset, unilaterality, poor vision at the time of diagnosis, and undergoing numerous procedures. ${ }^{18,19}$ The treatment of the condition is usually surgical with angle surgery, trabeculotomy, and goniotomy being among the most commonly 
performed procedures in these patients. ${ }^{20}$ The proportion of children who respond to medical treatment is generally lower than it is in the adult population. ${ }^{21}$

There is a relative lack of data on pediatric glaucoma in the region, and in Lebanon specifically. This paper aimed to report the characteristics of the affected population in Lebanon and to compare results with previously published studies in terms of demographics, surgical interventions, outcomes, and prognostic factors.

\section{Materials and Methods}

This was a retrospective chart review of pediatric glaucoma patients who presented to the ophthalmology department at the American University of Beirut Medical Center (AUBMC) over a span of 19 years (from January 1, 2000, to January 31, 2019). This list was gathered from hospital medical records using both surgery billing codes (trabeculectomy, goniotomy, trabeculectomy, and glaucoma shunt surgery) and ICD 9 codes. The study was approved by the Institutional Review Board (IRB) at the American University of Beirut and consent was waived. Furthermore, it abided by the principles stated in the Declaration of Helsinki. Demographic variables included age, gender, country of origin, and family history of glaucoma. Visit parameters recorded included date, age at presentation and first surgery, type of glaucoma and laterality, IOP and method of assessment, visual acuity, cup-to-disk ratio, current medications, surgical procedures, and complications. Parents of patients who reported themselves to be related were marked as consanguineous. Additionally, family history was marked positive if at least one family member was diagnosed with glaucoma. Most patients underwent sequential examinations under anesthesia where IOP was measured with a Tonopen XL (Reichert, Depew, New York, USA) or a Perkins tonometer (Clement Clarke, UK). They also underwent anterior segment examination, gonioscopy, axial length, and corneal diameter measurements and when possible, fundoscopy and retinoscopy. This information would complement any missing information from the clinic visit (largely due to the difficulty of examining a crying/agitated child).

Primary outcomes included: adequate IOP defined as a value $<21 \mathrm{~mm} \mathrm{Hg}$ (with or without medication) at the end of the follow-up period and good visual outcome defined as a best-corrected visual acuity (BCVA) of 20/50 or better. Angle surgery (goniotomy/ trabeculotomy) was considered successful if the respective eye did not require re-operation. Anisometropia was defined as an interocular refractive error difference of $\geq 3$ diopters for myopia, $\geq 1.5$ diopters for hyperopia, and $\geq 2$ diopters for astigmatism.

\section{Analysis}

Our chart review revealed a total of 40 patients (64 eyes) that had a follow-up of at least 1 year. Glaucomas were classified, as per the World Glaucoma Association, ${ }^{22}$ into either primary or secondary glaucomas. Primary glaucoma included PCG and juvenile open angle glaucoma (JOAG). Secondary glaucoma included aphakic and uveitic glaucoma and glaucoma due to non-acquired conditions such as Sturge-Weber syndrome and aniridia. Surgical procedures and medical therapies were identified and tallied. Surgical procedures included trabeculotomy, trabeculectomy with mitomycin C (MMC), cyclophotocoagulation, and Ahmed tube insertion while medical therapy included beta-blockers, prostaglandins, alpha agonists, and topical/oral carbonic anhydrase inhibitors. Trabeculotomy was the first line treatment for most patients with PCG in our center, unless canal of Schlemm could not be identified. ${ }^{23}$ In those cases, trabeculectomy (if eye was too small for tube shunt placement) or glaucoma shunt surgery (Ahmed tube) was performed. Cyclophotocoagulation using a diode laser to partially destroy the ciliary body was performed in eyes with worse visual prognosis or when surgical interventions failed to control IOP. ${ }^{24}$ Sight-threatening complications of these procedures include hypotony, choroidal detachment, intraocular hemorrhage, retinal detachment, and endophthalmitis. ${ }^{25}$

\section{Statistical Analysis}

The extracted data were entered into SPSS V25 (IBM SPSS Statistics for Windows, Version 25.0. Armonk, NY: IBM Corp., USA). Means and standard deviations were reported for demographic variables and eye examination parameters in addition to the number of medications and surgeries. As we only had three eyes that were diagnosed with JOAG, these were eliminated from our analyses as they are not numerous enough to be representative of the disease. A $p$ value of 0.05 or less was considered statistically significant.

\section{Results}

\section{Demographic and Presenting Variables}

Demographic variables and baseline characteristics of the studied eyes are reported in Table 1. Forty patients ( 64 eyes) were included: 29 eyes (45.3\%) had a primary form of glaucoma, while $35(54.7 \%)$ had secondary glaucoma. The most common diagnosis in the primary group was PCG, representing all of the primary cases included in this analysis. The most common type in the secondary group was aphakic glaucoma (45.7\%). Other types of secondary glaucoma included uveitic glaucoma (8.6\%), post-vitrectomy glaucoma (5.7\%), and glaucoma due to Peter's anomaly (5.7\%), aniridia (5.7\%), and

Table 1: Demographics and baseline characteristics

\begin{tabular}{lll}
\hline Type of glaucoma & Primary congenital glaucoma & Secondary glaucoma \\
\hline$N$ (patients/eyes) & $19 / 29$ & $21 / 35$ \\
Gender ratio (male:female) & $1.9: 1$ & $1: 1.2$ \\
Family history of glaucoma, $N(\%)$ & $4(13.8)$ & $10(28.6)$ \\
Parent consanguinity, $N(\%)$ & $10(34.5)$ & $0(0.0)$ \\
Bilateral involvement, $N(\%)$ & $20(69.0)$ & $28(80.0)$ \\
Follow-up duration, years (mean \pm SD) & $5.6 \pm 3.9$ & $7.8 \pm 5.2$ \\
Age at presentation, years (mean \pm SD) & $1.6 \pm 2.9[2$ days-10 years $]$ & $4.7 \pm 5.4[2$ days-17 years] \\
Age at first surgery, years (mean \pm SD) & $0.6 \pm 0.5$ & $3.4 \pm 5.2$ \\
IOP at presentation, mm Hg (mean \pm SD) & $31.9 \pm 13.5$ & $23.5 \pm 6.5$ \\
Initial C/D ratio (mean $\pm S D)$ & $0.4 \pm 0.2$ & $0.4 \pm 0.3$ \\
\hline
\end{tabular}


anterior segment dysgenesis (28.6\%). The follow-up duration for PCG and secondary glaucoma eyes was $5.6 \pm 3.9$ and $7.8 \pm 5.2$ years, respectively. More than half ( $54.7 \%)$ of all pediatric glaucoma eyes belonged to males. Fifty-six eyes (87.4\%) were those of Lebanese patients, whereas the remainder belonged to either Syrian (6.3\%) or Iraqi (6.3\%) individuals. Four eyes (13.8\%) in the PCG group had a positive family history of glaucoma, while in the secondary group this value was $28.6 \%$. Although $34.5 \%$ of primary glaucoma eyes came from individuals born to consanguineous parents; none of the secondary glaucoma patients had consanguineous parents. Patients with PCG were found to present earlier than those with secondary glaucoma and were operated on at a younger age. They also presented with a higher IOP than their secondary glaucoma counterparts $(31.9 \pm 13.5$ vs $23.5 \pm 6.5 \mathrm{~mm} \mathrm{Hg}, p=0.005)$. Initial cup-to-disk (C/D) ratio was $0.4 \pm 0.2$ and $0.4 \pm 0.3$, for PCG and secondary glaucoma eyes, respectively.

\section{Outcomes}

With regards to our primary outcomes (Table 2), $85.9 \%$ of all eyes achieved an IOP of $21 \mathrm{~mm} \mathrm{Hg}$ or less at the end of their follow-up period. In the PCG group, this value was $89.7 \%$ while in secondary glaucoma it was $82.9 \%$. Only $44.4 \%$ of all eyes had a final visual acuity of $20 / 50$ or better with the PCG group having a more favorable visual outcome than the secondary group (59.1 vs $34.4 \%$, respectively). Angle surgery failure rates were 5.7 and $10.3 \%$ for secondary and PCG eyes, respectively. Table 3 portrays the final visit parameters for PCG and secondary glaucoma groups. The IOP at the final visit was around $17 \mathrm{~mm} \mathrm{Hg}$ for both groups. logMAR visual acuity in the PCG group was $0.4 \pm 0.4$ and in the secondary group, it was $0.5 \pm 0.4$. Eighteen patients $(42.9 \%)$ had anisometropia: 8 with unilateral disease and 10 with bilateral involvement.

Table 2: Primary and secondary outcomes

\begin{tabular}{llll}
\hline & $\begin{array}{l}\text { Pediatric } \\
\text { glaucoma } \\
\text { (total) }\end{array}$ & $\begin{array}{l}\text { Primary } \\
\text { congenital } \\
\text { glaucoma }\end{array}$ & $\begin{array}{l}\text { Secondary } \\
\text { glaucoma }\end{array}$ \\
\hline $\begin{array}{l}\text { Final IOP } \leq 21 \\
\mathrm{~mm} \mathrm{Hg}(n, \%)\end{array}$ & $55 / 64(85.9)$ & $26 / 29(89.7)$ & $29 / 35(82.9)$ \\
$\begin{array}{l}\text { Final visual acuity } \geq \\
20 / 50\left(n^{*}, \%\right)\end{array}$ & $24 / 54(44.4)$ & $13 / 22(59.1)$ & $11 / 32(34.4)$ \\
$\begin{array}{l}\text { Failed angle surgery, } \\
N(\%)\end{array}$ & $5 / 64(7.8)$ & $3 / 29(10.3)$ & $2 / 35(5.7)$ \\
\hline
\end{tabular}

*Not all eyes had a numerical vision at the end of the follow-up period

Table 3: Final visit parameters

\begin{tabular}{llc}
\hline Type of glaucoma & $\begin{array}{l}\text { Primary congenital } \\
\text { glaucoma }\end{array}$ & $\begin{array}{l}\text { Secondary } \\
\text { glaucoma }\end{array}$ \\
\hline $\begin{array}{l}\text { IOP at end of the follow-up } \\
\text { period (mean } \pm \text { SD, mm Hg) }\end{array}$ & $16.5 \pm 6.9$ & $17.0 \pm 5.6$ \\
$\begin{array}{l}\text { Final C/D (mean } \pm \text { SD) } \\
\text { logMAR final visual acuity } \\
\text { (mean } \pm \text { SD) }\end{array}$ & $0.4 \pm 0.3$ & $0.6 \pm 0.2$ \\
$\begin{array}{l}\text { Mean number of surgeries } \pm \text { SD } \\
\text { (mean } \pm \text { SD) }\end{array}$ & $1.5 \pm 0.9$ & $0.5 \pm 0.4$ \\
$\begin{array}{l}\text { Surgery complication rate }(n, \%) \\
\begin{array}{l}\text { Need for non-glaucoma surgery } \\
\text { (n,\%) }\end{array}\end{array}$ & $1(3.4)$ & $1.5 \pm 1.2$ \\
$\begin{array}{l}\text { Number of medications at end of } \\
\text { the follow-up period (mean } \pm \text { SD) }\end{array}$ & $0.7 \pm 1.3$ & $3(8.6)$ \\
\hline
\end{tabular}

The mean number of surgeries was 1.5 for both groups. Table 4 demonstrates the number of eyes that underwent different glaucoma surgeries. Twenty-eight PCG and 13 secondary glaucoma eyes underwent trabeculotomies. All trabeculectomy procedures were done in patients with secondary glaucoma. Nine cyclophotocoagulation procedures were carried out in the secondary glaucoma group while only one was performed in the primary group. The number of Ahmed glaucoma valve implants was 7 and 11, for PCG and secondary glaucoma groups, respectively.

Table 5 shows the number of surgeries done per eye for each type of glaucoma. Overall, $83.6 \%$ of eyes underwent at least one glaucoma surgery. In PCG, the largest group (65.5\%) underwent one surgery per eye, while in secondary glaucoma, the largest group (31.4\%) did not undergo any surgeries. The only reported surgical complication in both groups was self-resolving hyphema (reported 1 and 3 times, for the PCG and secondary group, respectively). A number of study eyes also underwent several non-glaucoma surgeries including penetrating keratoplasty (PKP, $n=6$ ), Descemet's membrane endothelial keratoplasty (DMEK, $n=1)$, strabismus surgeries $(n=3)$, lensectomies $(n=8)$, and IOL insertions $(n=4)$.

With regards to the number of medications, secondary glaucoma eyes were taking $2.2 \pm 1.5$ medications at the end of their follow-up period while for primary glaucoma this value was $0.7 \pm 1.3$. Beta-blockers (40.3\%), prostaglandins (35.8\%), and topical carbonic anhydrase inhibitors (37.3\%) were more commonly prescribed than alpha agonists (17.9\%) and oral carbonic anhydrase inhibitors (4.5\%).

Looking at parameters affecting the first primary outcome (final $\mathrm{IOP} \leq 21 \mathrm{~mm} \mathrm{Hg}$ ): in the PCG group, the mean number of surgeries was significantly lower in the group with better IOP control $1.4 \pm$ 0.7 vs $3.0 \pm 1.0 \mathrm{~mm} \mathrm{Hg}$, respectively, $p=0.001$ ); however, this was not observed in the secondary glaucoma group. Regarding visual outcome: in the secondary glaucoma group, the average age at first surgery of those achieving good vision (final BCVA of 20/50 or better) was lower ( $0.6 \pm 0.4$ vs $4.5 \pm 5.8$ years, respectively, $p$ $=0.033)$, with a higher proportion of bilateral disease $(p=0.049)$. The number of surgeries in the group with good vision was also significantly lower $(0.7 \pm 0.9$ vs $1.9 \pm 1.2$, respectively, $p=0.01)$. No associations between final visual outcome and clinic parameters were observed in the PCG group.

\section{Discussion}

Our study aimed at describing the characteristics of pediatric glaucoma in a university hospital in Lebanon, while reporting on final visual outcomes and IOP control. We reviewed 64 eyes, $45.3 \%$ had a primary glaucoma diagnosis and the rest had secondary

Table 4: Number of eyes undergoing various types of glaucoma surgeries

\begin{tabular}{lcc}
\hline Type of surgery & $\begin{array}{l}\text { Primary congenital } \\
\text { glaucoma, } n(\%)\end{array}$ & $\begin{array}{l}\text { Secondary } \\
\text { glaucoma, } n(\%)\end{array}$ \\
\hline $\begin{array}{l}\text { Trabeculotomy } \\
\text { Trabeculectomy }+\end{array}$ & $28(96.6)$ & $13(37.1)$ \\
mitomycin C & $0(0.0)$ & $5(14.3)$ \\
$\begin{array}{l}\text { Ahmed glaucoma valve } \\
\text { implant }\end{array}$ & $7(24.1)$ & $11(31.4)$ \\
Cyclophotocoagulation & $1(3.4)$ & $9(25.7)$ \\
\hline
\end{tabular}


Table 5: Number of surgeries per eye

\begin{tabular}{lcrrrl}
\hline & \multicolumn{5}{c}{ Number of surgeries per eye } \\
\cline { 2 - 6 } & $0(\%)$ & $1(\%)$ & $2(\%)$ & $3(\%)$ & $4(\%)$ \\
\hline Primary congenital glaucoma & $0(0.0)$ & $19(65.5)$ & $8(27.6)$ & $2(6.9)$ & $0(0.0)$ \\
Secondary glaucoma & $11(31.4)$ & $9(25.7)$ & $7(20.0)$ & $7(20.0)$ & $1(2.9)$ \\
Pediatric glaucoma (all) & $11(17.2)$ & $28(43.8)$ & $15(23.4)$ & $9(14.1)$ & $1(1.6)$ \\
\hline
\end{tabular}

glaucoma. Our mean follow-up duration was 6.60 years. In the PCG group, there was a greater proportion of parent consanguinity compared to the secondary glaucoma group. Also, patients with PCG presented at a younger age and were operated on earlier than their secondary glaucoma counterparts. Furthermore, they had a significantly higher IOP at presentation. Regarding final outcomes, $85.9 \%$ of all studied eyes had good IOP control, while $44.4 \%$ of them achieved a final visual acuity of $20 / 50$ or better. Final IOP and cup-to-disk ratios did not differ between groups; however, the final number of medications was higher in the secondary group. Surgeries were distributed differently in the two groups: eyes with PCG underwent more trabeculotomies while secondary glaucoma eyes had more cyclophotocoagulation procedures done. The most commonly performed procedure overall was trabeculectomy (55.4\%), followed by Ahmed glaucoma valve implantation (24.3\%). All eyes in the PCG group underwent at least one surgery, while over $30 \%$ of eyes in the secondary group did not undergo any surgeries and were controlled medically.

Primary congenital glaucoma is believed to be the most frequent type of childhood glaucoma. ${ }^{26}$ Our study revealed an almost equal distribution of eyes with respect to these two types of glaucoma (29 eyes with PCG vs 35 with secondary glaucoma). This is in contrast with other studies looking at pediatric glaucoma in referral centers where the largest group was PCG. ${ }^{27}$ Several factors may have influenced the increased proportion of secondary glaucoma at our center. First, our center is a referral center and secondary glaucoma usually presents with multiple other comorbidities that may prompt referral. Second, this may also be due to the high levels of consanguinity in our population compared to other populations, ${ }^{28}$ and the genetic basis of diseases that cause secondary glaucoma such as anterior segment dysgenesis ${ }^{29}$ and post-congenital cataract surgery (aphakic glaucoma). ${ }^{30}$

Age at presentation in PCG eyes followed a similar trend to what was previously reported of presenting earlier than secondary glaucoma but the actual value was larger than what has been previously published. Our mean age at presentation was 1.6 years as opposed to $79 \%$ of cases presenting within 12 months of life in a British study ${ }^{2}$ and a median age diagnosis of 0.5 years by Saavedra et al. ${ }^{27}$ Due to the earlier presentation, it naturally follows that age at first surgery was earlier in PCG.

Presenting IOP in the PCG group was higher than that of secondary glaucoma (31.9 and $23.5 \mathrm{~mm} \mathrm{Hg}$, respectively). This is in contrast with what was previously published in a Saudi Arabian study that showed no significant differences between the two groups at baseline. ${ }^{31}$ Saavedra et al. reported a higher initial IOP in secondary glaucoma patients with both median values being lower than our means (16 and $20.5 \mathrm{~mm} \mathrm{Hg}$, for PCG and secondary glaucoma groups, respectively). However, the initial difference in our population did not seem to affect eventual IOP control. It has previously been reported that IOP at presentation carried no significant association with final IOP at 1 year. ${ }^{2}$ With regards to medications, PCG eyes had fewer medications at follow-up possibly reflecting the better control and prognosis of glaucoma in this group with surgical treatment. ${ }^{32} \mathrm{~A}$ similar trend was demonstrated by Saavedra et al. in a study carried out in Colombia which revealed that over $25 \%$ of PCG eyes were medication-free at the end of the follow-up period while in the secondary group this value was at $7.5 \% .^{27}$

The visual prognosis in children with glaucoma is correlated with the type of the associated ocular pathology..$^{17}$ In a review paper, Beck reported that PCG had the best visual prognosis, with $52-79 \%$ having a visual acuity of 20/50 or better. ${ }^{17}$ Glaucomas associated with congenital ocular anomalies and secondary glaucomas had a worse visual prognosis, with $30-50 \%$ of eyes noted to be $20 / 50$ or better. ${ }^{3,17,32-34}$ This study similarly demonstrated the better visual prognosis of PCG eyes compared to secondary glaucoma eyes (59.1 vs $34.4 \%$ of eyes achieving a final visual acuity of $20 / 50$ or better in PCG vs secondary glaucoma groups, respectively). Another study from Canada revealed a similar pattern in PCG eyes $(77.3 \%$ visual acuity equal $20 / 50$ or better with good IOP in all) compared to secondary glaucoma eyes. ${ }^{32} \mathrm{~A}$ study in the US population further reinforced the previous findings. ${ }^{18}$

In our region, similar trends have been reported regarding IOP control as well: a study done in the West Bank on 118 PCG eyes showed that $69 \%$ of them had good IOP control at the end of 43.9 months, off medications. ${ }^{10}$ Interestingly in our study, the number of eyes reaching good IOP control was quite higher: $86.6 \%$ of all studied eyes with or without meds with no significant differences between groups. The inclusion of patients controlled on medications in our definition may have led to the higher reported percentage. With regards to angle surgery outcomes, studies have portrayed similar success rates for trabeculectomy. ${ }^{34}$ Fulcher et al. reported a 92.3 and $85.7 \%$ success rate for trabeculectomy in primary infantile glaucoma and secondary glaucoma patients, respectively. ${ }^{34}$ Our results were comparable for PCG (89.7\%) and secondary glaucoma (94.3\%).

Our results also revealed that in the PCG group, an increased number of surgeries was associated with poor IOP control. Moreover, in secondary glaucoma, a high presenting IOP was associated with poor IOP control at the end of the follow-up period. With regards to final visual acuity, it was shown that at a later age at first surgery, unilateral disease, and multiple surgeries were associated with a poor BCVA $(<20 / 50)$. Our results are in concordance with previous studies. Khitri et al. previously reported that pediatric glaucoma patients with the unilateral disease were 5 times more likely to have a poor visual outcome (BCVA < 20/200)..$^{18}$ Moreover, those who underwent $>2$ surgeries were 32 times more likely to have visual impairment. $^{18}$

To our knowledge, this is the longest follow-up of patients with PCG and secondary glaucoma in the region at $6.6 \pm 4.7$ years (24 months in a study from Israel ${ }^{16}$ and 43.9 months in West Bank and $\mathrm{Gaza}^{10}$ ) comparing both types of pediatric glaucoma. Further 
strengths lie in the fact that our chart review was comprehensive with mostly complete data. Limitations included a relatively small sample size, which might have underpowered our study. Our work was also retrospective and depended mostly on paper charts; therefore, it is theoretically possible that some visits were missed. A few of our patients were preverbal at the end of their follow-up and did not have numerical visual acuity, and therefore were not included in computing final visual acuity.

\section{Conclusion}

Our study showed that PCG presented earlier and with a higher IOP than secondary glaucoma. These eyes were also operated on earlier. Both groups demonstrated good IOP control at the end of the follow-up period with PCG showing a better visual prognosis. Secondary glaucoma eyes required a higher number of medications at their final visit, reflecting the poorer IOP control of this group. When it came to surgeries, both groups underwent trabeculotomies most frequently. Prognostically, in the PCG group, an increased number of surgeries was associated with poor IOP control. In the secondary group, increased age at first surgery, unilateral disease, and multiple surgeries conferred a poor visual outcome.

\section{Clinical Significance}

Literature regarding the characteristics of pediatric glaucoma, especially in the Middle East, is scarce. Our study sheds light on the presentation of different types of pediatric glaucoma and their outcomes, namely IOP control and visual acuity.

\section{ACKnowledgments}

This study was approved by the Institutional Review Board (IRB) at the American University of Beirut.

\section{References}

1. Kipp MA. Childhood glaucoma. Pediatr Clin North Am 2003;50(1):89104. DOI: 10.1016/s0031-3955(02)00110-4.

2. Papadopoulos M, Cable N, Rahi J, et al. Investigators tBES. The British infantile and childhood glaucoma (BIG) eye study. Investigat Ophthalmol Visual Sci 2007;48(9):4100-4106. DOI: 10.1167/iovs.061350.

3. Taylor RH, Ainsworth JR, Evans AR, et al. The epidemiology of pediatric glaucoma: the Toronto experience. J Am Associat Pediat Ophthalmol Strabis 1999;3(5):308-315. DOI: 10.1016/S1091-8531(99)70028-5.

4. Qiao CY, Wang LH, Tang X, et al. Epidemiology of hospitalized pediatric glaucoma patients in Beijing Tongren hospital. Chin Med J (Engl) 2009;122(10):1162-1166.

5. Moschos MM, Nitoda E, Fenzel I, et al. Prognostic factors of pediatric glaucoma: a retrospective study. Int Ophthalmol 2019;39(2):359-373. DOI: 10.1007/s10792-018-0819-0.

6. Hoguet A, Grajewski A, Hodapp E, et al. A retrospective survey of childhood glaucoma prevalence according to childhood glaucoma research network classification. Indian J Ophthalmol 2016;64(2):118123. DOI: 10.4103/0301-4738.179716.

7. Mandal AK, Chakrabarti D. Update on congenital glaucoma. Indian J Ophthalmol 2011;59(Suppl 1):S148-S157. DOI: 10.4103/03014738.73683.

8. Tabbara KF, Badr IA. Changing pattern of childhood blindness in Saudi Arabia. Br J Ophthalmol 1985;69(4):312-315. DOI: 10.1136/bjo.69. 4.312.
9. Tadmouri GO, Nair P, Obeid T, et al. Consanguinity and reproductive health among Arabs. Reprod Health 2009;6(1):17. DOI: 10.1186/17424755-6-17.

10. Elder MJ. Congenital glaucoma in the West Bank and Gaza Strip. Br J Ophthalmol 1993;77(7):413-416. DOI: 10.1136/bjo.77.7.413.

11. Sivadorai $P$, Cherninkova $S$, Bouwer $S$, et al. Genetic heterogeneity and minor CYP1B1 involvement in the molecular basis of primary congenital glaucoma in Gypsies. Clin Genet 2008;74(1):82-87. DOI: 10.1111/j.1399-0004.2008.01024.x.

12. Al-Haddad C, Abdulaal M, Badra R, et al. Genotype/phenotype correlation in primary congenital glaucoma patients in the Lebanese population: A pilot study. Ophthalmic Genet 2016;37(1):31-36. DOI: 10.3109/13816810.2014.924015.

13. Gonzalez FJ. The molecular biology of cytochrome P450s. Pharmacol Rev 1988;40(4):243-288.

14. Bar-Yosef U, Levy J, Elbedour K, et al. Congenital glaucoma: CYP1B1 mutations in Israeli Bedouin kindreds. J Glaucoma 2010;19(1):35-38. DOI: 10.1097/IJG.0b013e3181a98b6f.

15. deLuise VP, Anderson DR. Primary infantile glaucoma (congenital glaucoma). Surv Ophthalmol 1983;28(1):1-19. DOI: 10.1016/00396257(83)90174-1.

16. Levy J, Carmi R, Rosen S, et al. Primary congenital glaucoma presenting within the first three months of life in a Bedouin population: prognostic factors. J Glaucoma 2005;14(2):139-144. DOI: 10.1097/01.ijg.0000151685.12033.04.

17. Beck AD. Diagnosis and management of pediatric glaucoma. Ophthalmol Clin North Am 2001;14(3):501-512. DOI: 10.1016/s08961549(05)70248-0.

18. Khitri MR, Mills MD, Ying G-S, et al. Visual acuity outcomes in pediatric glaucomas. J Am Associat Pediat Ophthalmol Strabis 2012;16(4): 376-381. DOI: 10.1016/j.jaapos.2012.05.007.

19. Chaudhary RS, Gupta A, Sharma A, et al. Long-term functional outcomes of different subtypes of primary congenital glaucoma. Br J Ophthalmol 2020;104(9):1288-1292. DOI: 10.1136/ bjophthalmol-2019-315131.

20. Scuderi G, lacovello D, Pranno F, et al. Pediatric glaucoma: a literature's review and analysis of surgical results. Bio Med Res Int 2015;2015:393670. DOI: 10.1155/2015/393670.

21. Sacchi M, Lizzio RAU, Villani E, et al. Medical management of pediatric glaucoma: lessons learned from randomized clinical trials. Graefe's Archive Clin Experim Ophthalmol 2020. 258(8):1579-1586. DOI: 10.1007/s00417-020-04767-9.

22. Childhood glaucoma the 9 th consensus report of the World Glaucoma Association, In: Weinreb RN, Grajewski AL, Papadopoulos M, et al., ed., Amsterdam, The Netherlands: Kugler Publications; 2013.

23. Nguyen $\mathrm{V}, \mathrm{Ab}$ Interno Trabeculectomy and Trabeculotomy. Accessed 29/12/2020, 2020. https://eyewiki.aao.org/Ab_Interno_ Trabeculectomy_and_Trabeculotomy.

24. Dastiridou Al, Katsanos A, Denis P, et al. Cyclodestructive procedures in glaucoma: a review of current and emerging options. Adv Ther 2018;35(12):2103-2127. DOI: 10.1007/s12325-018-0837-3.

25. Vijaya L, Manish P, Ronnie G, et al. Management of complications in glaucoma surgery. Indian J Ophthalmol 2011;59(Suppl1):S131-S140. DOI: 10.4103/0301-4738.73689.

26. Walton DS. Primary congenital open angle glaucoma: a study of the anterior segment abnormalities. Trans Am Ophthalmol Soc 1979;77:746-768.

27. Saavedra C, Rios HA, Belalcazar S, et al. Characteristics of pediatric glaucoma in a Latin American reference center. J Curr Glaucoma Pract 2020;14(1):10-15. DOI: 10.5005/jp-journals-10078-1267.

28. Barbour B, Salameh P. Consanguinity in Lebanon: prevalence, distribution and determinants. J Biosoc Sci 2009;41(4):505-517. DOI: $10.1017 / \mathrm{s} 0021932009003290$.

29. Reis LM, Semina EV. Genetics of anterior segment dysgenesis disorders. Curr Opin Ophthalmol 2011;22(5):314-324. DOI: 10.1097/ ICU.0b013e328349412b. 
30. Kirwan C, Lanigan B, O'Keefe M. Glaucoma in aphakic and pseudophakic eyes following surgery for congenital cataract in the first year of life. Acta Ophthalmol 2010;88(1):53-59. DOI: 10.1111/j.17553768.2009.01633.x.

31. Alanazi FF, Song JC, Mousa A, et al. Primary and secondary congenital glaucoma: baseline features from a registry at King Khaled eye specialist hospital, Riyadh, Saudi Arabia. Am J Ophthalmol 2013;155(5):882-889. DOI: 10.1016/j.ajo.2012. 12.006 .
32. Barsoum-Homsy M, Chevrette L. Incidence and prognosis of childhood glaucoma: a study of 63 cases. Ophthalmology 1986;93(10):1323-1327. DOI: 10.1016/S0161-6420(86)33569-3.

33. Morgan KS, Black B, Ellis FD, et al. Treatment of congenital glaucoma. Am J Ophthalmol 1981;92(6):799-803. DOI: 10.1016/s00029394(14)75633-8.

34. Fulcher T, Chan J, Lanigan B, et al. Long-term follow up of primary trabeculectomy for infantile glaucoma. $\mathrm{Br} J$ Ophthalmol 1996;80(6):499-502. DOI: 10.1136/bjo.80.6.499. 